\title{
MR-guided focused ultrasound (MRgFUS) ablation for non-spinal osteoid osteoma treatment: a prospective multicenter evaluation
}

\author{
D Geiger ${ }^{*}$, A Napoli, A Conchiglia, A Bazzocchi, M Mastantuono, U Albisinni, C Masciocchi, C Catalano \\ From 2nd European Symposium on Focused Ultrasound Therapy \\ Rome, Italy. 10-11 October 2013
}

\section{Background}

Purpose of this study was to evaluate technical success, complications and one year follow-up clinical success for non-spinal painful osteoid osteomas treated with MR-guided Focused Ultrasound (MRgFUS).

\section{Materials and methods}

This IRB approved prospective multicenter study was performed between May 2010 and April 2012. Thirty consecutive patients (M:21, F:9; Age range: 10-47; Mean age: $25 \pm 16$ ) have been enrolled at three different university centers for non-spinal painful osteoid osteomas and treated (29 lesions) using MRgFUS (3.0-T/1.5-T GE Discovery MR 750/450, Milwaukee, USA equipped with InSightec ExAblate 2100, Tirat Carmel, ISR). Lesions were previously diagnosed on imaging basis, including ce-dynamic MR (Gd-BOPTA, Bracco, Milan, Italy). Mean number of sonications and energy deposition has been recorded. Technical success was evaluated immediately after treatment for complications (skin burn, neurovascular and tendon or ligament injuries). Twelvemonth observation period followed to evaluate clinical success rate, recurrence and long-term complications. Clinical success was determined on the basis of pain reduction using visual analog scales (VAS); baseline and post-treatment scores statistical difference was calculated (paired T-test).

\section{Results}

Twenty-nine non-spinal osteoid osteomas have been successfully treated using MR-gFUS. Mean sonication number was $7 \pm 3$ and mean delivered acoustic energy $1180 \pm 736 \mathrm{~J}$. At 12 month follow-up complete clinical success was observed in 26 patients (26/29, 90\%; Mean VAS:0 \pm 0 ). Partial treatment was observed in 3 patients $(3 / 29,10 \%$; Mean VAS: $5 \pm 0)$, whom were treated in the early phase of the learning curve and subsequently underwent CT-gRFA (2/3) or open surgery (1/3). At clinical evaluation, pain score values showed a significant reduction $(\mathrm{p}<0.001)$ between baseline (Mean VAS: $8 \pm 1$ ) and post-treatment (Mean VAS: $1 \pm 2$ ). No complications have been observed during, immediately after treatment and at one-year follow-up.

\section{Conclusion}

Results of this prospective multicenter study, suggest that MR guided focused ultrasound may be considered an effective and safe alternative approach in non-spinal osteoid osteoma interventional management. Complete clinical success rate of $90 \%$ was demonstrated without adverse events.

Published: 10 December 2014

doi:10.1186/2050-5736-2-S1-A24

Cite this article as: Geiger et al:: MR-guided focused ultrasound (MRgFUS) ablation for non-spinal osteoid osteoma treatment: a prospective multicenter evaluation. Journal of Therapeutic Ultrasound 2014 2(Suppl 1):A24.

Sapienza University of Rome, Italy 